\title{
Study on the Campus Smart Card System based on SaaS
}

\author{
Jianwen Feng \\ Department of Computer Science and Engineer, Hanshan Normal University, Chaozhou, China \\ fjw@gmail.com
}

Keywords: Smart Card, SaaS, Integration, Configurability.

\begin{abstract}
The smart card system has become an essential part of the digital campus. Recently the solutions based on SaaS in China have become popular for the advantages of less upfront investment, good service quality and convenience maintainability. But in colleges and universities there are many challenges. In this paper, we discussed the solutions for three challenges: personalized demand, system integration and information utilization. Firstly, it was suggested that different users should select the private cloud or public cloud mode. Next, the campus smart card system model based on SaaS was proposed. The model with layered architecture has good configurability, flexibility and integration metrics. Then, several methods were put forward to solve the integration between the card systems with other information systems. In addition, the IA theory was applied in the information retrieval, and the intelligent analysis examples of the card business data were discussed. At last we considered five kinds of configurability: data, function, workflow, portal and smart card, and a configurability management system based on metadata was presented.
\end{abstract}

\section{Introduction}

Using smart card as the carrier of information, the campus smart card system (CSCS) is relying on the campus network to provide identification, campus management and other application services with the Internet of things, the Internet, software engineering and database technology[1, 2]. Since the beginning of twenty-first Century, experiencing the hardware architecture, application architecture, software architecture and service architecture stages, the CSCS has become the infrastructure and necessary management tool of digital campus[3]. But the excellent solutions need a lot of supports such as sufficient fund, advanced information technology, perfect information management method and strong team. These let most of the colleges stop. Recently the solutions based on SaaS have become popular for its advantages of less upfront investment, good service quality and convenience maintainability y [4]. But in colleges and universities there are still many challenges. This paper aims at three challenges: personalized needs, system integrated management and information resource utilization.

To do these, in section 2, the application modes for different universities being classified, the campus smart card system model based on SaaS is proposed, and its logical architecture, integrated architecture, data architecture and function design are discussed. In section 3, we focus on the solutions meeting with the problems: information resources integration, information resources utilization and personalize configurability

\section{Model Design}

Application Mode. The As of 2014 July, the higher school (not including the independent college) in China has a total of 2542, including 2246 universities and Colleges and 296 adult institutions. And in Guangdong Province, there are 142 universities and colleges, including 4 ' 211 ' and 2 '985' universities only [5]. Because of the limited resources, most of the higher schools purchase the products to establish the smart card system, but not enough to upgrade and improve the system, which can't really play the role of the smart card system. SaaS can just solve the problem of limited resources. 
According to the demands and characteristics of different high schools, two application patterns of CSCS are as follows:

Private cloud scheme: faces with the universities having more than one campus, big scale and sufficient fund.

Public cloud scheme: suitable for small and middle scale colleges with less fund and weak technology or management strength.

Model Design. The high school users usually have three kinds of individual demands: software functions, business rules and information integration. To meet with these, a system with configurability and feasibility is advised.

Most of SaaS solutions ordinary use the maturity model, whose maturity features include: scalability, configurability and multi-user efficiency[6, 7]. In this case the logical architecture of model uses the maturity model III focusing on scalability and configurability. Next we propose an according model of CSCS based on SaaS in Figure 1.

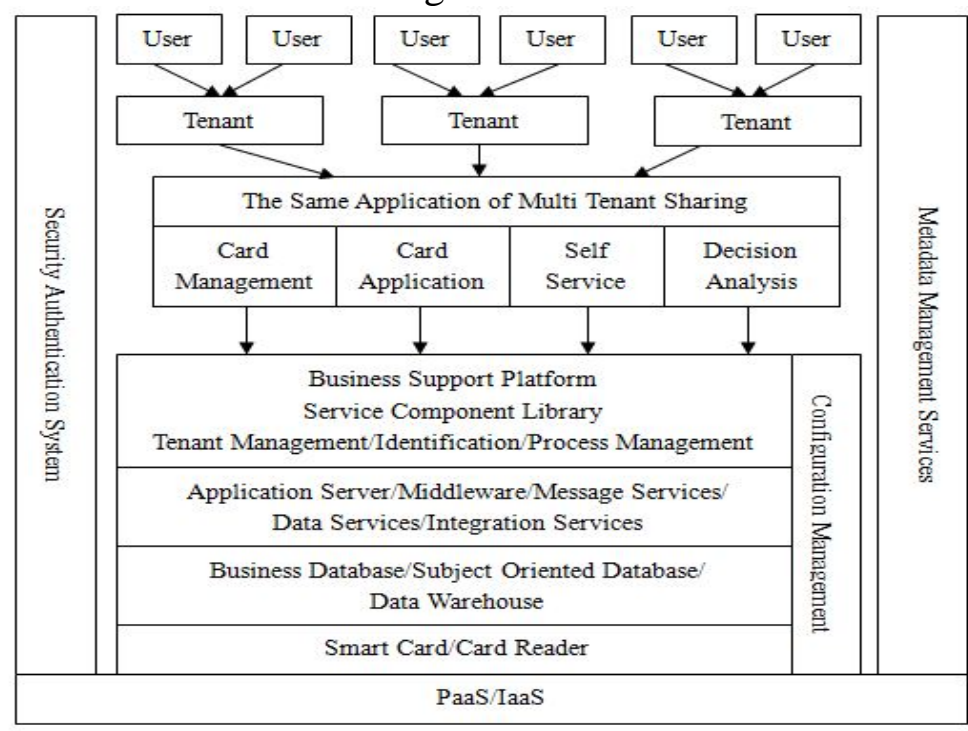

Fig. 1 The Model of CSCS based on SaaS

The model can obtain a better balance between resource utilization and cost so that can be used for private cloud or public cloud early stage. In latter stage we transition to maturity model IV for the large-scale users to promote the multi-user efficiency. In addition, the model uses the service combination and call technique to achieve the need of information resources integration. The technique is based on SOA which uses XML scheme as service presentation, ESB bus as message transmitter and SOAP as call interface[8].

As shown in figure 1, the model with multilayer: Business Layer, Business Support Layer, Data Layer and Hardware Layer. And there are security system, configuration management and metadata management services. In the Data Layer, data architecture uses the scheme of a shared database combining multi isolation data structures[9]. The shared database can realize the resource sharing between different users, while the method of isolation data structures uses the configurable metadata, authorization and security management to provide every user unique experiences and functions. In addition the subject oriented database and data warehouse with the integration service in business support layer can service for the information resource utilization. In general, the model focuses on configuration and integration characteristics in order to solve the personalization and resource integration requirements of the high schools.

Function Design. The whole system includes two parts: business support center and application service center. The functions of business support center consist of the following:

Data management: data storage scheme maintenance, data backup, import and export management, personalized data management

Announcement and news management

User management: user data management, user join and quit

Billing management 
Safety management: user authorization and identification

Monitoring: monitor user behavior, control unreasonable behavior, monitor system performance

Service integration: service definition and call management.

Then application service center offer various application services for every users. The services integrating in the SaaS platform by Web Services come from the products of different software services and the modification of old systems.

\section{Methods}

Integration of Information Resources. In recent years, with its popularity, the CSCS has change from application system toward service system and Information Resource Center. More and more application systems have been integrated into the CSCS. Because information integration is the basis of information utilization and business applications, so the topic of information resources integration has become the key of CSCS.

First of all it is important for establishing a global data environment to guide the integration of information resources. The environment includes the data file, business database, subject oriented database and information retrieval system [10]. It is suggested that the application environment consists of the former two things, and the information resource utilization environment has the latter two.

Second, the system model should adopt hierarchical structure. For example, the campus card system in FuDan University is divided into four layers: database layer, management layer, application layer and hardware layer, which can not only access hardware equipment but share and synchronize data with other data centers [11].

At last as shown in Table 1, there are many methods of information integration for different application situations [12-16].

Table 1 Information integration methods of CSCS

\begin{tabular}{|c|c|}
\hline Situation & Method \\
\hline Source codes are available & Modify the source codes \\
\hline Data export or import are available & Use the interfaces \\
\hline $\begin{array}{l}\text { Interfaces must be meet with the } \\
\text { techniques standards }\end{array}$ & Develop the access function by the standards \\
\hline Above are not available & $\begin{array}{l}\text { Use monitoring and intercepting techniques, such as Win API } \\
\text { functions, monitoring memory and intercepting the network data } \\
\text { packages }\end{array}$ \\
\hline Flexibility is & Use web services and XML techniques \\
\hline Data synchronization is necessary & $\begin{array}{l}\text { Use the temporary data table, logs, stored procedure and trigger } \\
\text { methods }\end{array}$ \\
\hline
\end{tabular}

Utilization of Information Resource. Utilization of information resources means information resource retrieval and data analysis.

On the information retrieval, the existing systems mostly execute simple inquiry and statistics, without the support by reasonable information retrieval method and information management theory. Next, in the help of the Information Architecture (IA) theory and method, we make the information resource retrieval mechanism as shown in Figure 2 to build the multi-application retrieval system [17]. 


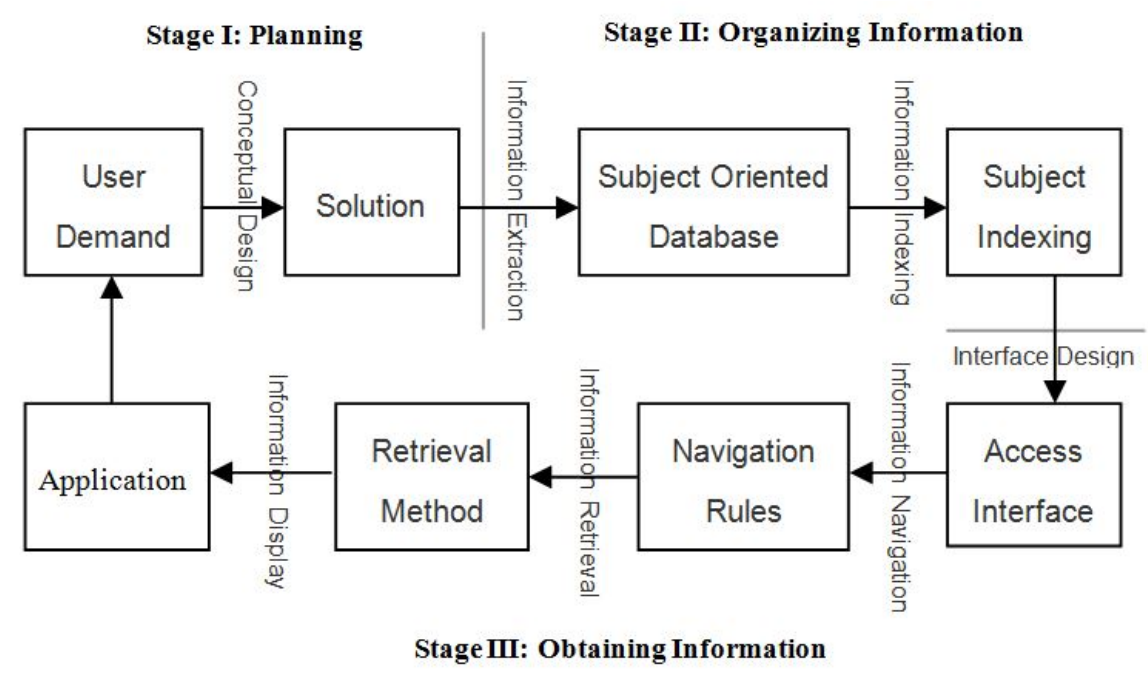

Fig. 2 the process of the CSCS based on IA

The whole process is divided into three stages: planning, organizing information and obtaining information. The first stage is to make a plan meeting with the user demands. In organizing information stage, the subject oriented database is built by information extraction and the various subjects are indexed. The information processed will be obtained through an access interface under the control of navigation rules and retrieval methods in the last stage.

Now on the data analysis, some researches and applications have begun to appear. In Fudan University, the college students' consumption level analysis has been carried out for management decision support in the help of the sharing database established independent of CSCS[11]. Similarly, East China Normal University has made the students' water and food consumption analysis by the association rules and clustering methods[18]. In addition, the CSCS is applied in the teaching supervision and teaching facilities management of the Medical University of Chongqing experimental[19].

However the above of the data analysis application are simple, not up to the requirements of commercial products. There are no applications in CSCS based on SaaS environment. It is advised that introducing the techniques and methods of data mining, machine learning and statistics into the CSCS applications help the school administration to carry out the intelligent application of cross section and personal.

Configurability. The maturity model III in Figure 1 with the same application sharing by multi-tenants method is good for the service providers less cost and more convenient management. But in the CSCS environment, not only each school tenants will have individual needs, and each of the users groups of tenant may propose unique requirements. According to the internal management mode and process, the users will put forward various personalized needs about the system functions, data, workflow and interface etc.

So the CSCS must meet this goal. We need a model with configurability metrics, which in general includes the data configurability, application function configurability, business processes configurability and operation interface configurability[20]. Beyond that, the configurability of smart card is very important in CSCS based on SaaS since there are various solutions for different smart card techniques.

At different levels, we focus on different configurability. Take the model in Figure 1 as an example. We focus on data configurability in the data layer, operation interface configurability in the portal layer, application function configurability in the business layer, business processes configurability in the business support layer and smart card configurability in the hardware layer.

A configuration management system based on metadata and the model driven architecture (MDA) is to manage all configurability. The parameters of configurability are defined so that the relations and dependences among metadata of configurability can be established. The system model is shown in Figure 3. 


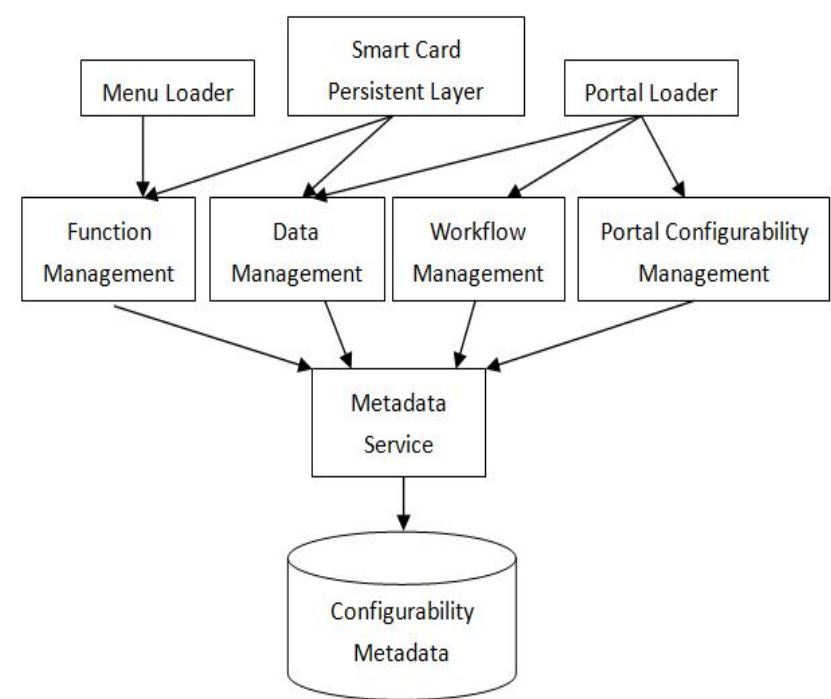

Fig. 3 the configuration management system based on metadata

There are four configurability management parts in this system: function management, data management, workflow management and portal configurability management. They all access the metadata through the metadata services. When a user signing in, the portal loader will show the access interface set by the portal configurability management, data management and workflow management, and according to the function management, the menu loader displays the function menu in the portal interface. If the user logins with a smart card, the persistent layer can identify the kind of card, and limit the personalize functions and data by the predefined rules.

\section{Summary}

Facing three challenges about CSCS based on SaaS, a layering model with scalability and configurability is put forward, and the solutions about information integration and utilization are discussed in detail. Then the configurability of smart card is introduced into the configuration management for the first time. In future, the security, robustness and execute performance must be paid more attention to.

At present, most of the colleges have begun to focus on application services rather than hardware-centric. The SaaS taking the software as a service is consistent with this tendency of China education informatization. But because of policy, management ideas, technology and other factors, the application based on SaaS progress slowly. It is advised that the education administration should encourage informatization reform of colleges and universities to improve the benefit of running a school. And scientific research institutes and companies should also actively study the common problem in the education industry, so as to make the successful combination of cloud computing and education informatization.

\section{Acknowledgement}

This paper is supported by Science Research Foundation of HanShan Normal University under Grant No.LT201101.

\section{References}

[1] Wolfgang, R., and Wolfgang, E., 2004, Smart Card Handbook, John Wiley and Sons.

[2] Liu, J., 2008, "Design and application of digital campus card system," Journal of Guangxi University for Nationalities (8), p. 85. 
[3] Jianwen, F., Feng, L., and Xuan, L., "Current situation and development of China Campus Card System," Proc. Artificial Intelligence and Education (ICAIE), 2010 International Conference on, pp. 469-474.

[4] Zhendong, X., Jincheng, W., and Dandan, T., 2014, "Study on the construction of public transportation card interconnection platform based on Guangdong Hong Kong and Macao," Journal of Golden Card Project (1).

[5] China, M. o. E. o. t. P. s. R. o., 2014, "List of 2014 national higher school in China (as of July 9, 2014)," http://www.moe.edu.cn.

[6] Chong, F., and Carraro, G., 2006, "Architecture Strategies for Catching the LongTail."

[7] Chunying, F., 2008, "To build the SaaS trusted platform," Journal of Programmer (8), p. 5.

[8] Baolan, Y., 2009, "Design and Application of Campus card platform based on SOA," Journal of Jilin Normal University (2), p. 134.

[9] Bryant, B., 2009, "Multi-tenant database design."

[10] Xianhua, J., 2005, "Information integration based on Virtual Database,"Master, Chongqing University.

[11] Jiabin, X., Shujuan, L., and Yong, B., 2009, "Campus card construction based on software-centric and the Fudan University case study," Journal of Sun Yet-sun University (3), p. 175.

[12] Sathyan, J., and Shenoy, K., "Realizing unified service experience with SaaS on SOA," Proc. Communication Systems Software and Middleware and Workshops, 2008. COMSWARE 2008. 3rd International Conference on, pp. 327-332.

[13] Mietzner, R., Leymann, F., and Papazoglou, M. P., "Defining Composite Configurable SaaS Application Packages Using SCA, Variability Descriptors and Multi-tenancy Patterns," Proc. Internet and Web Applications and Services, 2008. ICIW '08. Third International Conference on, pp. 156-161.

[14] Jianwen, F., 2006, "Application of Win32 hook in the card in the system," Journal of Fujian computer (9), p. 28.

[15] Yueguo, C., and Chunjing, C., 2004, "Data integration review," Journal of Computer science, 31(5).

[16] Chuanwen, X., and Weiyun, X., 2007, "Design and implementation of campus smart card system based on Middleware," Journal of Computer engineering and design (4), p. 1723.

[17] Jianwen, F., Feng, L., and Xiaochun, Y., "A Model of the Campus Smart Card System based on IA," Proc. Asia-Pacific Youth Conference on communication Technology 2010, Scientific Research Publishing, USA.

[18] Zhicheng, H., 2009, "Design and development of system of campus card decision based on Data Mining," Journal of Modern computer (12), p. 175.

[19] Qiuyu, X., Hong, M., and TingTing, T., 2014, "Mining application of campus card system in experimental teaching management," Journal of Experiment science and technology, 11(4).

[20] Mietzner, R., and Leymann, F., "Generation of BPEL Customization Processes for SaaS Applications from Variability Descriptors," Proc. Services Computing, 2008. SCC '08. IEEE International Conference on, pp. 359-366. 